\title{
PRONÓSTICO DE LA DEMANDA MENSUAL DE ELECTRICIDAD CON SERIES DE TIEMPO
}

\section{¿. Víctor Daniel GiL Vera ${ }^{1}$}

\section{RESUMEN}

La alta volatilidad de los precios de la electricidad ha motivado a diseñar a investigadores y académicos modelos que permitan pronosticar la demanda de la misma en el corto, mediano y largo plazo. Este trabajo presenta un modelo de pronóstico de la demanda mensual de electricidad basado en series de tiempo, el cual toma como base los valores de la demanda de energía eléctrica en el Sistema Interconectado Nacional (SIN) de Colombia en el período 2008-2014. Se concluye que las series de tiempo aplicadas al pronóstico de la demanda de electricidad permiten predecir con un alto grado de exactitud demandas futuras de energía eléctrica (GWh), información que puede generar ventajas a productores, distribuidores y grandes consumidores a la hora de establecer estrategias, optimizar su operación y realizar contratos bilaterales.

PALABRAS CLAVE: Demanda mensual de electricidad; mercados de energía; modelos de pronóstico; series de tiempo.

\section{MONTHLY FORECAST OF ELECTRICITY DEMAND WITH TIME SERIES}

\section{ABSTRACT}

The high volatility of electricity prices has motivated researchers and academics to design models that will enable the forecast of electricity demand in short, medium and long terms. This paper presents a model for forecasting the monthly electricity demand based on time series. The model uses the electricity demand values of Colombia's National Interconnected System (NIS) for the 2008-2014 period as its base. It was concluded that the time series applied to the electricity demand forecast enable a high accuracy level of prediction of future electricity demands (GWh), information which can lead to advantages for producers, distributors and large consumers when establishing strategies, streamlining operations and reaching bilateral agreements.

KEYWORDS: Energy markets; Forecasting models; Monthly electricity demand; Time series.

1 Ingeniero Administrador - Universidad Nacional de Colombia. MSc en Ingeniería de Sistemas - Universidad Nacional de Colombia. Estudiante de Doctorado en Ingeniería de Sistemas - Universidad Nacional. Magíster en Administración de Empresas. Universidad Católica Luis Amigó, Medellín, Antioquia, Colombia.

Autor de correspondencia: Gil Vera, V.D. (Víctor Daniel). Tel.: 3218637163

Correo electrónico: victor.gilve@amigo.edu.co
Historia del artículo:

Artículo recibido: 18-I-2016 / Aprobado: 13-X-2016

Disponible online: 30 de febrero de 2017

Discusión abierta hasta abril de 2018 


\section{PRONÓSTICO DA DEMANDA MENSAL DE ELECTRICIDADE COM SÉRIES DE TEMPO}

\section{RESUMO}

A alta volatilidade dos preços da eletricidade tem motivado a desenhar a pesquisadores e académicos modelos que permitam pronosticar a demanda da mesma no curto, médio e longo prazo. Este trabalho apresenta um modelo de pronóstico da demanda mensal de eletricidade baseado em séries de tempo, o qual toma como base os valores da demanda de energia elétrica no Sistema Interconectado Nacional (SIN) da Colômbia no período 2008-2014. Conclui-se que as séries de tempo aplicadas ao pronóstico da demanda de eletricidade permitem predizer com um alto grau de exatidão demandas futuras de energia elétrica (GWh), informação que pode gerar vantagens a produtores, revendedores e grandes consumidores à hora de estabelecer estratégias, otimizar sua operação e realizar contratos bilaterais.

PALAVRAS-CHAVE: Demanda mensal de eletricidade; Mercados de energia; Modelos de prognóstico; Séries de tempo.

\section{INTRODUCCIÓN}

La gran importancia de la electricidad en la vida del hombre ha motivado a un gran número de empresas a crear modelos estructurales o basados en costeo para obtener estimaciones de sus costos futuros (Velásquez y Souza, 2007). El pronóstico de la demanda de electricidad tiene asociados una serie de factores entre los que se destacan: la alta volatilidad de los precios de la energía, relaciones no lineales complejas, incertidumbres, limitaciones humanas, políticas organizacionales, problemas metodológicos, experiencias limitadas, aspectos regulatorios, entre otros, que la convierten en una tarea de alta complejidad (Velásquez et al., 2007). En la mayoría de los mercados de energía a nivel mundial las decisiones operativas y estratégicas de los participantes toman como referente principal los pronósticos estimados con anterioridad. Entre las principales razones que justifican la necesidad de crear un modelo que sirva para pronosticar la demanda mensual de electricidad se encuentran:

- En el mercado de corto plazo los productores de energía necesitan predicciones para formular estrategias de comercialización, y optimizar su programa de generación; en el mediano plazo, para negociar contratos bilaterales; y en el largo plazo, para la toma de decisiones relacionadas con el portafolio de activos de generación, la adquisición de nuevas plantas, y el abandono de plantas existentes (Velásquez et al., 2007).

- En el corto y mediano plazo los distribuidores y grandes consumidores necesitan predicciones para optimizar su operación, para negociar adecuadamente en el mercado de corto plazo, y para realizar contratos bilaterales (Velásquez et al., 2007).

- El administrador del mercado requiere de buenos pronósticos para realizar un adecuado manejo y planeación del mismo (Velásquez et al., 2007).

El objetivo de este trabajo es presentar un modelo basado en series de tiempo para el pronóstico de la demanda mensual de electricidad, el cual se construyó y validó tomando como base los valores de la demanda de energía eléctrica en el SIN de Colombia en el período 2008-2014. Para la construcción del modelo se utilizaron series de tiempo, definidas como una extensión de valores en la misma escala, indexados por un parámetro de tiempo similar (Brillinger, 2015), las cuales se constituyen como una técnica de fácil implementación y de resultados confiables para el pronóstico de la demanda de electricidad, al igual que otras técnicas de pronóstico como lo son las redes neuronales artificiales (RNA) o los modelos de regresión lineal. En estas, los datos básicos y los parámetros son funciones (Brillinger, 
2015). Los datos empleados corresponden a los valores de la demanda de energía eléctrica en el SIN de Colombia en el período 2008-2014, los cuales fueron obtenidos de la compañía XM S.A E.S.P., entidad encargada de operar el Sistema Interconectado Nacional (SIN) de Colombia y de administrar el Mercado de Energía Mayorista (MEM). El modelo fue desarrollado e implementado en el software estadístico R.

El artículo está organizado de la siguiente forma: después de la introducción, se presenta una contextualización general del mercado eléctrico colombiano, las nociones básicas de series de tiempo, la metodología empleada para la construcción del modelo, los resultados obtenidos y la validación. Finalmente, se concluye que las series de tiempo aplicadas a la predicción de la demanda de electricidad permiten realizar aproximaciones con un alto grado de exactitud, lo que permite generar ventajas a productores, distribuidores y grandes consumidores a la hora de establecer estrategias, optimizar su operación y realizar contratos bilaterales.

\section{MERCADO ELÉCTRICO \\ COLOMBIANO}

En la década de los 80, el sector eléctrico colombiano entró en crisis, al igual que otros de países de América Latina. El deterioro en su desempeño se debió, especialmente, al subsidio de tarifas y a la politización de las empresas estatales (Botero et al., 2008). Los grandes proyectos de generación, llevados a cabo con sobrecostos y atrasos considerables, condujeron a que el sector se convirtiera en una gran carga para el Estado (Botero et al., 2008). Colombia liberalizó el sector eléctrico y su mercado mayorista de electricidad en 1995, con un diseño similar al modelo del mercado inglés de la época. Desde el inicio de la operación del Mercado Eléctrico Mayorista (MEM) de Colombia, el 20 de julio de 1995, se establecieron dos mecanismos de negociación de electricidad: los contratos no estandarizados y la negociación directa en el mercado spot de corto plazo (Velásquez, Gil, y Franco, 2015). En la actualidad, las bases legales están estipuladas en las Leyes 142 y 143 de 1994 (Dyner et al., 2012). Con el fin de garantizar la eficiencia y confiabilidad en la prestación del servicio, se vio en la necesidad de reestructurar el sector basándose en la experiencia de países pioneros como el Reino Unido (Rendón et al., 2011). A continuación, se presenta una breve descripción de estas leyes:

- La Ley 142 (Ley de servicios públicos domiciliarios), pretende garantizar la eficiencia y calidad en la prestación de los servicios, mediante la regulación de los monopolios existentes y la promoción de la competencia (Régimen Legal de Bogotá D.C, 1994; Sandoval, 2004). Además, busca una mayor participación del sector privado y la ampliación en la cobertura de la prestación de los servicios, donde el Estado garantice la prestación del servicio mediante la planeación, regulación y control (Rendón et al., 2011).

- La Ley 143 (Ley eléctrica), establece los criterios por medio de los cuales se rige la prestación del servicio de electricidad y por tanto el funcionamiento del sector, y crea algunos entes encargados de la dirección, planeación, regulación y supervisión de cada una de las actividades que conforman el eslabón de la cadena productiva (Régimen Legal de Bogotá D.C, 1994; Sandoval, 2004).

Los principales órganos están conformados por instituciones y entidades de administración y operación, las cuales se describen en las secciones 1.1 y 1.2 , respectivamente.

\subsection{Instituciones del sector}

- Comisión de Regulación de Energía y Gas (CREG): se encarga de regular el sector energético, de electricidad y gas, con el fin de prestar un servicio más eficiente y de mejor calidad (Rendón et al., 2011). Su función es garantizar al usuario final la obtención de mayores beneficios, a través de la disminución de costos, con el objetivo de obtener el máximo aprovechamiento de los recursos (Rendón et al., 2011). 
- Superintendencia de Servicios Públicos Domiciliarios (SSDP): organismo de carácter técnico, sus principales funciones son controlar y vigilar las entidades prestadoras de servicios públicos domiciliarios (Rendón et al., 2011).

- Unidad de Planeación Minero Energética (UPME): unidad administrativa especial adscrita al Ministerio de Minas y Energía. Su principal función es la elaboración de los planes de expansión referentes al sector eléctrico (Rendón et al., 2011).

\section{2. Órganos de Administración y operación}

- Centro Nacional de Despacho (CND): dependencia de XM, filial de Interconexión Eléctrica S.A (ISA), encargada de supervisar, controlar y planear la operación integrada de los recursos de generación, interconexión y trasmisión del sistema nacional con el objetivo de tener una operación económica, confiable y segura (Rendón et al., 2011).

- Administrador del Sistema de Intercambios Comerciales (ASIC): dependencia de XM, filial de Interconexión Eléctrica S.A (ISA), encargada de todo lo relacionado con el registro de fronteras comerciales y contratos de energía a largo plazo y todos los trámites que se requieran (Rendón et al., 2011).

- Liquidador y Administrador de Cuentas del Sistema de Transmisión Nacional (STN): dependencia de XM, filial de Interconexión Eléctrica S.A (ISA), que participa en la administración del Mercado de Energía Mayorista (MEM), encargada de facturar y liquidar los cargos relacionados con el uso de las redes del sistema interconectado nacional (Rendón et al., 2011).

El mercado eléctrico colombiano funciona como lo dictamina la Comisión de Regulación de Energía y Gas (CREG), la cual determina las reglas de operación y el funcionamiento para todos los participantes del mercado (Dyner et al., 2012). La prestación del servicio de energía eléctrica en Colombia al usuario final está compuesta por las actividades de generación, transporte, distribución y comercialización (Rendón et al., 2011). La primera y la última se aproximan a una estructura competitiva, aunque en la realidad son oligopolios; mientras que las dos intermedias están caracterizadas como monopolios naturales (Rendón et al., 2011). A continuación se describen las actividades mencionadas:

- Generación: consiste en la producción de energía eléctrica. El Estado debe garantizar que esta actividad se desarrolle por medio de una estructura competitiva, evitando el poder de mercado (Rendón et al., 2011).

- Transporte: se encarga del transporte de energía a altos niveles de voltaje a través del Sistema de Transmisión Nacional (STN), conjunto de líneas y subestaciones con equipos y transformadores con módulos de conexión que operan con tensiones entre 220 y 500 kW (Aguilar y Díaz, 2004; UPME, 2004).

- Distribución: se transforman los altos niveles de tensión (voltaje) de la energía para ser llevada al usuario final. En el país se lleva a través de Sistemas de Transmisión Regionales (STR) y los Sistemas de Distribución Locales (SDL) (Rendón et al., 2011).

- Comercialización: compra de la energía en el mercado mayorista para venderla a los usuarios finales. La estructura de esta actividad es competitiva. El papel del Estado principalmente consiste en garantizar la no concentración o la no existencia de poder de mercado (Rendón et al., 2011).

En la bolsa de energía, se transan los contratos de corto plazo (Gil, 2015). Las transacciones que se realizan en este mercado pueden ser, al igual que en los contratos bilaterales, entre generadores y comercializadores o entre algunos de los anteriores (Rendón et al., 2011). Los generadores presentan el día anterior un precio único para el día siguiente y declaran disponibilidad al Centro Nacional de Despacho (CND), que es el operador del mercado (Rendón et al., 2011). Por orden de mérito de menor precio se despachan las disponibilidades declaradas que sean necesarias para abastecer el pronóstico de demanda. La última unidad despachada 
necesaria para abastecer la demanda determina el precio marginal del sistema mediante el cual se remunera a todos los oferentes que resultaron despachados (Rendón et al., 2011).

El mecanismo de oferta se realiza por medio de una subasta de precio uniforme y los precios de oferta deben reflejar los costos variables de generación (Rendón et al., 2011). Así como en la gran mayoría de los mercados mayoristas, los demandantes actúan como agentes pasivos en el mercado. El CND recibe las ofertas de los generadores e inicia el pre despacho de energía basado en las proyecciones de demanda construidas a partir de: datos históricos, modelos de planeación y estudios dados por la Unidad de Planeación Minero Energética (UPME), sin considerar las restricciones del sistema; es decir, que no se toman en consideración los límites operativos de los elementos asociados al Sistema Interconectado Nacional (SIN) ni las indisponibilidades no declaradas de los mismos. Debido a la reestructuración del sector eléctrico colombiano, durante las dos últimas décadas, el comportamiento del precio de la energía eléctrica ha incrementado su volatilidad, reflejando el riesgo existente para los diferentes agentes que intervienen en el mercado (Botero et al., 2008).

\section{SERIES DE TIEMPO}

Los pronósticos son una herramienta que proporciona un estimado cuantitativo de la probabilidad de eventos futuros (Juárez, Zuñiga, Flores, y Partida, 2016). Una de las técnicas para hacerlo son las series de tiempo, utilizadas en una amplia gama de disciplinas: finanzas, transporte, mercados, biología, medicina, ingeniería, etc., para analizar el comportamiento de una variable en el tiempo y facilitar la toma de decisiones (Moreno-García, Jiménez-Linares, y Rodríguez-Benítez, 2017). Estas toman como base datos históricos para obtener una caracterización estadística de los enlaces existentes en el pasado y en el presente (Diebold, 2001). En las últimas dos décadas, el desafío de predecir el futuro mirando el pasado ha conducido a la creación de una variedad de algoritmos de predicción de series de tiempo (Egrioglu, E., Yolcu, U. y Aladag, C. 2015; Hyndman, R.J., Khandakar, Y 2008). Estos permiten considerar información temporal, estimar parámetros y proporcionar datos puntuales precisos sobre valores futuros (Lütkepohl, H. 2005; Pravilovic, Bilancia, Appice, y Malerba, 2017).

Una serie de tiempo es una sucesión de variables aleatorias ordenadas de acuerdo a una unidad de tiempo $, Y_{1} \ldots \ldots, T_{T}$. Estas son útiles para obtener pronósticos de valores futuros de la serie, con el fin de ayudar a tomar decisiones que tienen consecuencias importantes o para entender mejor el mecanismo de generación de los datos, que puede no ser claro inicialmente en una investigación (Giraldo, 2011). También son utilizadas para analizar el comportamiento dinámico de sistemas complejos (Zunino, Olivares, Bariviera, y Rosso, 2017).

Las series de tiempo se clasifican en estacionarias y no estacionarias. Las primeras se caracterizan por ser estables a lo largo del tiempo. En las series de tiempo no estacionarias la tendencia puede cambiar a lo largo del tiempo (Giraldo 2011). Los principales componentes de las series de tiempo: tendencia, estacionalidad y heterocedasticidad, indican el comportamiento periódico y a largo plazo de la serie. Dada una serie $T_{t}, t=1, \ldots . . ., T_{\text {, el }}$ Modelo Aditivo $Y_{t}$ consiste en asumir que se puede descomponer en tres componentes (Quintana y Jiménez, 2016). Ver Ecuación (1):

$$
Y_{t}=T_{t}+S_{t}+\varepsilon_{t}
$$

Donde $T_{t}$ es la tendencia, $S_{t}$ es la componente estacional y $\varepsilon_{t}$ es la componente de errores. Las componentes $T_{t}$ y $S_{t}$ son funciones de $t$ determinísticas y su evolución es perfectamente predecible (Giraldo, 2011). Una serie de tiempo tiene componente estacional de período $S$, si presenta patrones que se repiten con una frecuencia constante $(S)$, produciendo en ella un efecto periódico (Diebold, 2001). 


\section{METODOLOGÍA}

Para la construcción del modelo, se emplearon los valores de la demanda mensual de electricidad (GWh) del SIN de Colombia en el período 20082014, obtenidos de la compañía XM S.A E.S.P, entidad encargada de operar el SIN de Colombia y de admi- nistrar el MEM. Los valores mensuales de la demanda registrados en el año 2014 se emplearon para la validación del modelo. La Figura 1, presenta la tendencia mensual de la demanda de energía eléctrica en el SIN de Colombia en el período 2008-2013.

La Tabla 1, presenta los datos utilizados para la construcción del modelo:

Figura 1. GWh Vs. Tiempo

Fuente: elaboración propia

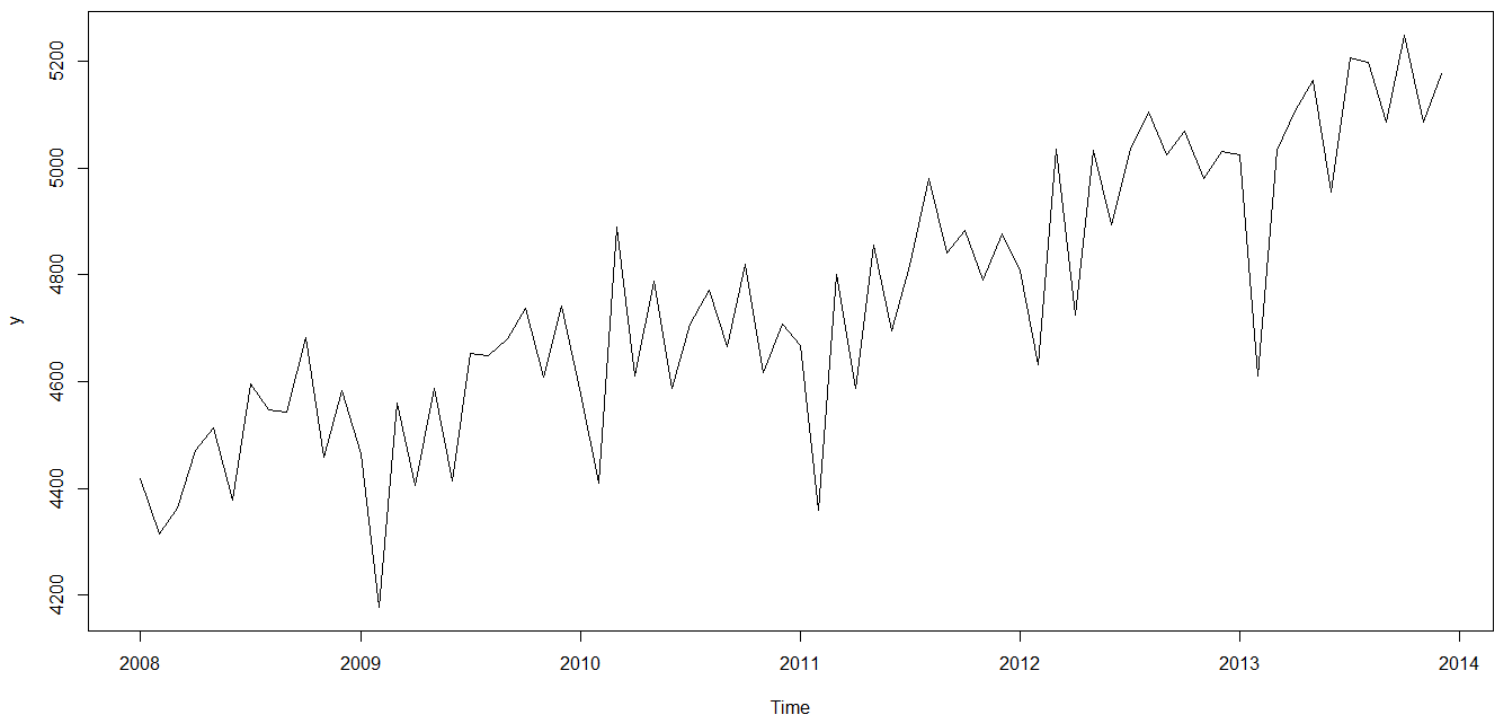

TABLA 1. DEMANDA MENSUAL DE ELECTRICIDAD EN COLOMBIA 2008-2014

FUENTE: XM S.A E.S.P.

Demanda Mensual de Electricidad (GWh)

\begin{tabular}{c|c|c|c|c|c|c|c|c|c|c|c|c}
\multirow{2}{*}{ Año } & Enero & Febrero & Marzo & Abril & Mayo & Junio & Julio & Agosto & Sept & Octubre & Noviembre & Diciembre \\
\hline 2008 & 4418,50 & 4314,8 & 4363,5 & 4470,3 & 4513,1 & 4377,9 & 4595,4 & 4546,6 & 4544 & 4682,5 & 4459,5 & 4583,5 \\
\hline 2009 & 4464,70 & 4177,2 & 4560,1 & 4406,1 & 4586,6 & 4414,3 & 4653,4 & 4649,4 & 4681,2 & 4737,3 & 4607,6 & 4741,2 \\
\hline 2010 & 4576,90 & 4409,5 & 4890,1 & 4610,6 & 4788 & 4587 & 4707 & 4772 & 4665 & 4819 & 4616 & 4707 \\
\hline 2011 & 4667,00 & 4359 & 4801 & 4587 & 4855 & 4694 & 4817 & 4979 & 4841 & 4883 & 4791 & 4876 \\
\hline 2012 & 4806,90 & 4631,4 & 5033,9 & 4724,7 & 5032,7 & 4893,7 & 5033,9 & 5104,9 & 5024,8 & 5069,6 & 4979,6 & 5031 \\
\hline 2013 & 5025,20 & 4609,7 & 5033 & 5106,3 & 5163,4 & 4955 & 5205,5 & 5196,5 & 5084,6 & 5249,1 & 5086 & 5176,4 \\
\hline 2014 & 5166,90 & 4902,2 & 5316,6 & 5168,9 & 5410,8 & 5218 & 5512,6 & 5418,9 & 5345,8 & 5461,3 & 5250,7 & 5397,4 \\
\hline
\end{tabular}


El modelo desarrollado fue construido con el software estadístico $\mathrm{R}$, entorno de programación para la computación y generación de gráficos estadísticos, compatible para una amplia variedad de plataformas: UNIX, Windows y MacOS (R Cran Project 2015), y distribuido bajo la licencia GNU GPL (General Public License) (Wikipedia 2015b). Para la construcción de los modelos se emplearon las siguientes librerías: Car, Forecast, Lmtest y Zoo. En este trabajo se desarrollaron tres modelos de series de tiempo aditivos con diferentes tendencias: lineal, cuadrática y cúbica. La Ecuación (2), presenta el modelo general de tendencia, donde $p$ es el orden del polinomio de la función de la componente de tendencia.

$$
T_{t}=\beta_{0}+\beta_{1} t+\beta_{2} t^{2}+\ldots+\beta_{p} t^{p}
$$

La Tabla 2, presenta el código empleado en cada uno de los modelos:

\begin{tabular}{|c|c|c|}
\hline \multicolumn{3}{|c|}{$\begin{array}{l}\text { TABLA 2. CÓDIGOS } \\
\text { FUENTE: ELABORACIÓN PROPIA }\end{array}$} \\
\hline $\begin{array}{l}\text { Tendencia } \\
\text { lineal }\end{array}$ & $\begin{array}{l}\text { Tendencia } \\
\text { cuadrática }\end{array}$ & Tendencia cúbica \\
\hline $\bmod =\operatorname{lm}(z \sim t+\mid t)$ & $\bmod 2=\operatorname{lm}(z \sim t+t 2+l t)$ & $\bmod 3=\operatorname{Im}(z \sim t+t 2+t 3+\mid t)$ \\
\hline summary(mod) & summary $(\bmod 2)$ & summary (mod3) \\
\hline $\mathrm{AIC}(\mathrm{mod})$ & $\mathrm{AIC}(\bmod 2)$ & $\mathrm{AIC}(\bmod 3)$ \\
\hline $\mathrm{BIC}(\bmod )$ & $\mathrm{BIC}(\bmod 2)$ & $B I C(\bmod 3)$ \\
\hline
\end{tabular}

El mejor modelo fue seleccionado según el principio metodológico y filosófico de la "Navaja de Ockham", también conocido como el principio de parsimonia o de economía, atribuido al fraile franciscano, filósofo y lógico escolástico Guillermo de Ockham (1280-1349), el cual establece que: "en igualdad de condiciones, la explicación más sencilla suele ser la correcta" (Wikipedia 2015a). La Tabla 3, presenta los valores de los parámetros y los deltas mensuales para cada uno de los modelos desarrollados:

\begin{tabular}{|c|c|c|c|}
\hline \multicolumn{4}{|c|}{$\begin{array}{l}\text { TABLA 3. PARÁMETROS. } \\
\text { FUENTE: ELABORACIÓN PROPIA }\end{array}$} \\
\hline \multirow{2}{*}{ Parámetros } & \multicolumn{3}{|c|}{ Modelo } \\
\hline & Lineal & Cuadrático & Cúbico \\
\hline$\beta 0$ & 4397,694 & 4490,91994 & 4482 \\
\hline$\beta 1$ & 11,0971 & 4,49365 & 5,618 \\
\hline$\beta 2$ & 0 & 0,07769 & 0,04492 \\
\hline$\beta 3$ & 0 & 0 & 0,000257 \\
\hline$\delta 1$ & $-75,9885$ & $-75,98852$ & $-73,97$ \\
\hline$\delta 2$ & $-333,1285$ & $-332,35164$ & $-330,5$ \\
\hline$\delta 3$ & 26,4029 & 27,80129 & 29,44 \\
\hline$\delta 4$ & $-116,7371$ & $-114,87259$ & $-113,4$ \\
\hline$\delta 5$ & 54,4086 & 56,58388 & 57,86 \\
\hline$\delta 6$ & $-129,5028$ & $-127,17218$ & $-126,1$ \\
\hline$\delta 7$ & 57,2429 & 59,57353 & 60,5 \\
\hline$\delta 8$ & 66,5029 & 68,67815 & 69,42 \\
\hline$\delta 9$ & $-13,2943$ & $-11,42975$ & $-10,86$ \\
\hline$\delta 10$ & 77,8086 & 79,20697 & 79,59 \\
\hline$\delta 11$ & $-92,06$ & $-91,28311$ & $-91,09$ \\
\hline R cuadrado & 0,9449 & 0,9626 & 0,9626 \\
\hline $\begin{array}{l}\text { R cuadrado } \\
\text { ajustado }\end{array}$ & 0,9355 & 0,9556 & 0,9551 \\
\hline
\end{tabular}

Los criterios utilizados para la selección del mejor modelo fueron: $\mathrm{R}$ cuadrado ajustado $\left(R_{a d j}^{2}\right)$ más grande, Criterio de Información Akaike (AIC) y Criterio de Información Bayesiano (BIC) más pequeños. A continuación, se presentan las respectivas ecuaciones de los criterios mencionados. Ver Ecuación (3), (4) y (5):

$$
\begin{aligned}
& R_{a d j}^{2}=1-\frac{(T-1) S S E}{(T-K) S S T} \\
& A I C=\frac{(T-k) S S E}{T(T-p)} e^{2 k / T} \\
& B I C=\frac{(T-k) S S E}{T(T-p)} T^{k / T}
\end{aligned}
$$

Donde $T$ es el número de períodos muestreados, $P$ es el número de parámetros del modelo y $K=$ $P+1$. La Tabla 4, presenta los valores de cada uno de los criterios mencionados para cada uno de los modelos desarrollados: 


\begin{tabular}{|c|c|c|c|}
\hline Indicador & Lineal & Cuadrático & Cúbico \\
\hline AIC & 985,0532 & 954,5419 & 956,354 \\
\hline $\mathrm{BIC}$ & 1019,085 & 991,0042 & 995,247 \\
\hline $\begin{array}{l}\text { R cuadrado } \\
\text { ajustado }\end{array}$ & 0,9355 & 0,9556 & 0,9551 \\
\hline
\end{tabular}

Se observa que el modelo cuadrático es el que tiene el mayor R cuadrado ajustado ( $\mathrm{R}=0.9556)$, el menor AIC $(954,5419)$ y el menor BIC $(991,0042)$, por lo cual es el que mejor se ajusta a las condiciones del modelo.

\section{VALIDACIÓN Y RESULTADOS}

Para la validación del modelo, se tomó como base la demanda mensual registrada en el año 2014. Para cada mes se calculó la demanda según los componentes del modelo y el porcentaje de error. La Tabla 5, presenta las demandas estimadas y reales (GWh) y el porcentaje (\%) de error.

\begin{tabular}{|c|c|c|c|c|}
\hline \multicolumn{5}{|c|}{ TABLA 5. VALIDACIÓN DEL MODELO. } \\
FUENTE: ELABORACIÓN PROPIA \\
Mes & $\begin{array}{c}\text { Deltas } \\
\text { mensuales } \\
\text { (GWh) }\end{array}$ & $\begin{array}{c}\text { Demanda } \\
\text { pronosticada } \\
\text { por el modelo } \\
\text { (GWh) }\end{array}$ & Real (GWh) & $\begin{array}{c}\text { Demanda } \\
\text { Rorcentaje } \\
\text { (\%) }\end{array}$ \\
\hline 73 & $-83,14893$ & 5133,19491 & 5166,9 & 0,66 \\
\hline 74 & $-335,41083$ & 4895,52098 & 4902,2 & 0,14 \\
\hline 75 & 18,46872 & 5264,11372 & 5316,6 & 1,00 \\
\hline 76 & $-120,54362$ & 5139,93979 & 5168,9 & 0,56 \\
\hline 77 & 42,05217 & 5317,49921 & 5410,8 & 1,75 \\
\hline 78 & $-137,26059$ & 5153,2753 & 5218 & 1,26 \\
\hline 79 & 34,50143 & 5340,25139 & 5512,6 & 3,23 \\
\hline 80 & 63,78824 & 5384,87749 & 5418,9 & 0,63 \\
\hline 81 & $-14,3835$ & 5322,17026 & 5345,8 & 0,44 \\
\hline 82 & 75,26955 & 5427,41304 & 5461,3 & 0,62 \\
\hline 83 & $-85,31928$ & 5282,53916 & 5250,7 & 0,60 \\
\hline 84 & 0 & 5383,69861 & 5397,4 & 0,25 \\
\hline
\end{tabular}

La Figura 2, presenta la serie de valores de la Demanda Real vs la Demanda Pronosticada por el modelo para el año 2014:

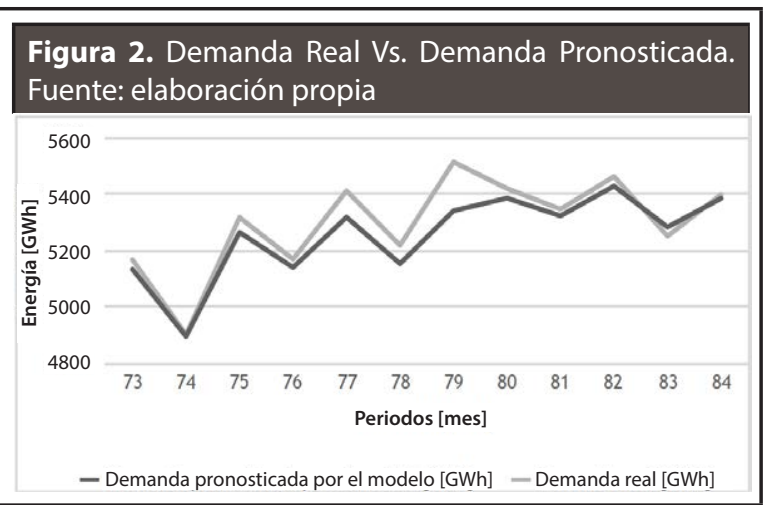

Algunos estadísticos básicos empleados para medir qué tan adecuado es el modelo para pronosticar la demanda mensual de electricidad fueron: Error Promedio de Pronóstico (ME), Error Promedio Absoluto de Pronóstico (MAE) y Error Cuadrático Medio de Pronóstico (MSE). Ver Ecuación (6), (7) y (8):

$$
\begin{gathered}
M E=\frac{1}{m} \sum_{i=0}^{m-1} e_{n+i}=-43.801 \\
M A E=\frac{1}{m} \sum_{i=0}^{m-1}\left|e_{n+i}\right|=33.705 \\
M S E=\frac{1}{m} \sum_{i=0}^{m-1}\left(e_{n+i}\right)^{2}=1136.033
\end{gathered}
$$

El MPE y el MAPE son estadísticos intuitivos y fáciles de interpretar puesto que representan el porcentaje promedio del error o el porcentaje absoluto promedio del error de los pronósticos y suministran una información más concreta sobre la precisión del pronóstico que el MSE. En el modelo seleccionado, el estadístico MAPE=0,915\% es inferior al MSIMP $=1,136 \%$, lo que indica que el modelo desarrollado reduce el porcentaje de error relativo en la predicción en un $0,45 \%$ con respecto al método simple, lo que garantiza el uso del modelo de predicción desarrollado. Ver Tabla 6:

Revista EIA Rev.EIA.Esc.Ing.Antioq / Universidad EIA 


\begin{tabular}{|c|c|}
\hline \multicolumn{2}{|l|}{ TABLA 6. RESULTADOS. } \\
FUENTE: ELABORACIÓN PROPIA \\
\hline Índice & Valor \\
\hline ME (GWh) & $-43,801$ \\
\hline MAE (GWh) & 33,705 \\
\hline MSE (GWh)2 & 1136,033 \\
\hline RMSE (GWh) & 33,705 \\
\hline MPE (\%) & $-0,814$ \\
\hline MAPE (\%) & 0,915 \\
\hline MSIMP (\%) & 1.36 \\
\hline
\end{tabular}

\section{CONCLUSIONES}

Pronosticar la demanda de la electricidad es una tarea de gran importancia para todos los agentes participantes del mercado eléctrico colombiano (administradores, generadores, distribuidores y grandes consumidores), ya que permite tener una visión futura del comportamiento del mercado, ayudan a establecer estrategias de comercialización, optimizar programas de generación y negociar contratos bilaterales beneficiosos. Entre los diferentes métodos y técnicas utilizadas para pronosticar la demanda de electricidad, las series de tiempo permiten obtener predicciones con un alto grado de exactitud. En este trabajo, se han desarrollado tres modelos utilizando series de tiempo aditivas con diferentes tendencias (lineal, cuadrática y cúbica). El modelo con tendencia cuadrática fue el modelo que mejor se ajustó a los criterios de selección: R cuadrado ajustado más grande, Criterio de Información Akaike (AIC) y Criterio de Información Bayesiano (BIC) más pequeños: $R_{a d j}^{2}=0,9556, A I C=954,5419$, $B I C=991,0042$. El porcentaje promedio de error de la diferencia entre los precios reales y los pronosticados no superan el 0,93\%, lo que indica que el modelo desarrollado presenta un alto nivel de acercamiento a los precios reales.

\section{REFERENCIAS}

Aguilar, A.; Díaz, J. (2004). Una visión del mercado eléctrico colombiano. Bogotá: Unidad de Planeación Minero Energética (UPME).

Botero, S.B; Cano Cano, J.A. (2008). Análisis de series de tiempo para la predicción de los precios de la energía en la bolsa de colombia. Cuadernos de Economía, 48, pp. $174-207$.

Brillinger, D.R. (2015). Time Series: General. International Encyclopedia of the Social y Behavioral Sciences, 2, pp.341-346. [Online] Disponible en: http://www. sciencedirect.com.ezproxy.unal.edu.co/science/article/pii/B9780080970868420842.

Diebold, F. (2001). Elements of forecasting 2nd ed. South y W. C. Publishing., eds., Australia.

Dyner, I.; Franco, C.J.; Arango, S. (2012). El mercado mayorista de electricidad colombiano 1st ed. C. F. de Minas, ed., Medellín: Universidad Nacional de Colombia.

Egrioglu, E.; Yolcu, U.; Aladag, C. (2015). Red neuronal artificial multiplicativo modelo neuronal recurrente para el pronóstico de series de tiempo no lineales. Proceso Neural. Lett., 41(2), pp. 249-258

Gil, V. D. (2015). Forecasting Electricity Demand for Small Colombian Populations. Cuaderno Activa, 7(7), 111120.

Giraldo, N. (2011). Modelado y Pronóstico de la Tendencia. pp. 17-29. [Online] Disponible en: http://www. medellin.unal.edu.co/ ndgirald/Archivos\%20Lectura/Archivos\%20curso\%20Series\%20EIO/Trabajo_No1_EstadisticaIII_sem01_12.pdf [Consultado 4 de marzo de 2016].

Hyndman, R.J.; Khandakar, Y. (2008). Serie de Tiempo automático de predicción: el paquete pronóstico para R. J. Stat. Software, 27 (3), pp. 1-22.

Juárez, A. C.; Zúñiga, C.A.; Flores, J.L.M.; Partida, D.S. (2016). Análisis de series de tiempo en el pronóstico de la demanda de almacenamiento de productos perecederos. Estudios Gerenciales, 32(141), 387-396.

Lütkepohl, H. (2005). Nueva Introducción al Análisis Temporal de Series Múltiples. Springer-Verlag Berlin Heidelberg.

Moreno-García, A.; Moreno-García, J.; Jiménez-Linares, L.; Rodríguez-Benítez, L. (2017). Time series represented by means of fuzzy piecewise lineal segments. 
Journal of Computational and Applied Mathematics, $318,156-167$

Pravilovic, S.; Bilancia, M.; Appice, A.; Malerba, D. (2017). Using multiple time series analysis for geosensor data forecasting. Information Sciences, 380, 31-52.

Quintana, M.J.G.; Jiménez, S.A.M. (2016). Modelos de series de tiempo aplicados a los expedientes de la Comisión de Derechos Humanos del Distrito Federal. Economía Informa, 398, 89-99.

R Cran Project (2015). The Comprehensive R Archive Network. p.1. [Online] Disponible en: https://cran.rproject.org/ [Consultado 24 de julio de 2015].

Régimen Legal de Bogotá (1994). Ley 143 de 1994 Nivel Nacional. [Online] Disponible en: https://www. minminas.gov.co/documents/10180/667537/ Ley_143_1994.pdf/c2cfbda4-fe12-470e-9d3067286b9ad17e [Consultado 3 de marzo de 2017].

Régimen Legal de Bogotá D.C. (1994). Ley 142 de 1994 Nivel Nacional. [Online] Disponible en: http://www. alcaldiabogota.gov.co/sisjur/normas/Norma1. jsp?i=2752 [Consultado 3 de marzo de 2017].

Rendón, J.G.; Hinestroza, A.G.; Moreno, L.S. (2011). Determinantes del precio de la energía eléctrica en el mercado no regulado en Colombia. Revista Ciencias Estratégicas., 19(26), pp. 225-246.

Sandoval, A.M. (2004). Monografía del sector de electricidad y gas colombiano: Condiciones actuales y retos futuros. pp. 27-45. [Online] Disponible en: https:// colaboracion.dnp.gov.co/CDT/EstudiosEconmicos/272.pdf.

UPME (2004). Una visión del mercado eléctrico colombiano. pp. 1-110.

Velásquez, J.; Souza, R. (2007) ¿Por qué es tan difícil obtener buenos pronósticos de los precios de la electricidad en mercados competitivos? Revista Cuadernos de Administración, 20(34), pp. 259-282.

Velásquez, J.D.; Gil, V.; Franco, C.J. (2015). An Overview of the Colombian Market for Standarized Derivatives of Energy Commodities. IEEE Latin America Transactions, 13(7), 2176-2182.

Wikipedia (2015a). Navaja de Ockham. pp. 1-3. [Online] Disponible en: http://es.wikipedia.org/wiki/Navaja_de_Ockham [Consultado 9 de junio de 2015].

Wikipedia (2015b). R: lenguaje de Programación., pp.1-3. [Online] Disponible en: http://es.wikipedia.org/ wiki/R_(lenguaje_de_programación) [Consultado 9 de junio de 2015].
Zunino, L.; Olivares, F.; Bariviera, A.F.; Rosso, O. A. (2017). A simple and fast representation space for classifying complex time series. Physics Letters A, 381(11), 1021-1028.

\section{PARA CITAR ESTE ARTÍCULO / TO REFERENCE THIS ARTICLE / PARA CITAR ESTE ARTIGO /}

Gil Vera, V.D. (2016). Pronóstico de la demanda mensual de electricidad con series de tiempo. Revista EIA, 13(26), juliodiciembre, pp. 111-120. [Online]. Disponible en: https://doi. org/10.24050/reia.v13i26.749 